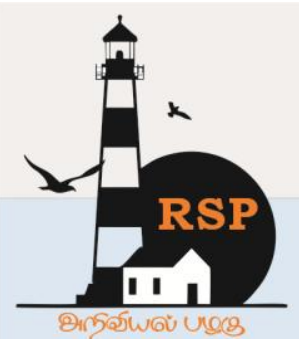

INTERNATIONAL RESEARCH JOURNAL ON ADVANCED SCIENCE HUB

Special Issue of First International Conference on Advancements in Management, Engineering and Technology (ICAMET 2020)

\title{
Conceptualizing the Importance of HR Analytics in Attrition Reduction
}

Rupa Chatterjee Das ${ }^{1}$, Dr. Anjana Devi S.C ${ }^{2}$

${ }^{1}$ Assistant Professor, Department of Management Studies, Dayananda Sagar Academy of Technology \& Management, Bangalore, Karnataka, India,

${ }^{2}$ Associate Professor, Department of Management Studies, Dayananda Sagar Academy of Technology \& Management, Bangalore, Karnataka, India,

rupa.c.das84@gmail.com ${ }^{1}$,anjanadevisc@gmail.com ${ }^{2}$

\begin{abstract}
The objective of the conceptual paper for analysing the opportunities and challenges of HR Analytics for reducing the attrition rate in the organization. The paper highlighted the important reasons of high attrition in different type of organizations and the effective approaches by the respective organizations for reducing attrition rate. Employee attrition is one of serious issues in the current corporate scenario as it impacts on the organizational image, values, culture, reputation, and job satisfaction. Moreover, high attrition is related to high cost for any organization. This research intended to explore employee attrition and finding out gaps in work and improve in those areas for reducing attrition accordingly. The sources of data used are both primary \& secondary. HR Managers of select organizations were requested to share the recent trends with the usage \& applications of HR Analytical tools. The data was collected by conducting personal interactions over telephones as primary source. Journals \& research articles in the domain of $H R$ analytics were used to get better insights. Effective recruitment structure, working environment and Employee Engagement is created. Though the prediction by HR Analytics is a challenging job but there are many methodologies which can change the look of the organization and $H R$ team can measure and predict the gap for attrition. This research paper explains the analytical process for reducing attrition and importance of utilization of HR Analytics.
\end{abstract}

Key Words- HR Analytics, Employee Attrition, Retention, Value-Culture-Job SatisfactionReputation, Recruitment, Job Structure.

\section{Introduction}

HR Analytics is the method of collecting and evaluating Human Resource data to improve an organization's culture, workers performance, retention etc. (Eric W. MacIntosh, Alison Doherty - 2009[5]). HR Analytics has the power of early prediction of the movement of the employees and organization. In the big organization where huge no of employees are working, HR Analytical tools or methodology are very much productive for providing the data-supported understanding on what is working fine and what is not so that organizations can make changes or improvements and plan more efficiently for the future (Sandeep Yadav, Aman Jain \& Deepti Singh-2018[1]).

The most used Analytical tools by organizations for their analytical works are as (e.g. [16]) -

1. $\mathbf{R}$ is one of the most common tools of HR analytics. It is most famous for statistical 
analysis and visualization. It can explore huge data sets. Like able to analyze and clean millions of rows of data.

2. Python is a programming language, and it is very much useful for substituting with $\mathrm{R}$. Mainly in the IT organizations use this programming language for the analytical work in the organization.

3. Excel is the most basic tool of HR Analytics. For any type of numerical and graphical calculations, we use this tool very frequently. This tool also quite common for all organization and HR team as well. Excel is most user-friendly tool for all employees.

4. Tableau is remarkably like Power BI in that it allows the accumulation and conception of various data sources. This tool founded in 2003 for research produced at Stanford University, the software has created identical position in the world. It is one of the best business intelligence tools in the current scenario.

5. SPSS another one of the most used tools of HR analytics in the social disciplines. It is very user-friendly approach; through this tool anyone can analyze the data without proper statistical knowledge as well. As it is very user friendly anyone wants to analyze data, they can do the same easily.

In this paper, made a sincere attempt to understand the significant of HR Analytics to deliver high performance to the organization. The first part reviews the previous work done by existing authors and discovers the gap for the research. Second portion highlighted the study objectives of the research. The last section explains about input process and output framework of effective parameters for reducing attrition in the organization. The last section provides implication of the effective tools and framework followed by conclusions and references.

\section{Literature Review}

In the literature review, collected differing viewpoints and assessments have given idea on the various factors of employee attrition. The highlighted statistics are the outcomes of concentrated literature review and the references helped to understand employee engagement and organizational structure, conceptualizing the importance of HR Analytics in attrition reduction.

\section{Sandeep Yadav (2018)}

Sandeep Yadav et.al (2018) in their research on "Early Prediction of Employee Attrition using Data Mining Techniques" have focused on the importance of data mining techniques to forecast the attrition patterns in employees.

Researchers have taken an IT Industry along with different departments of the organization for their research on prediction of employee attrition. Authors have used - Name of the employee, Number of Projects handled, Average Monthly Hours given, Job Satisfaction Level, Time spent in the organization (Years), Last Evaluation, Departments, Work mishap, Absent, Promotion \& Rewards last 5 years, compensation, salary level as a variables for measuring the reasons of attrition. Techniques as Data Pre-processing, Featuring Engineering, Data Modelling \& Comparison are employed the research Hot Encoding process is used to steely various department as IT, Accounting, Management, HR, Product Management, Sales, Support, Technical. Logistic Regression, SVM Model are usedfor getting the Categorization Results of Hot Programming Method.

The outcome of these study shows, Employee attrition can influence an organization in many disciplines like reputation, revenues, position in the market, and cost in terms of both time and money. So, during hiring if the organization able to take preventive measure that will help the organization to reduce attrition. It was understood that compensation, career development, promotions are not the only justifications at the back of the employee attrition. During hiring other aspects also, company needs to consider. And through this concept the firm can build dependable and correct models which can improve the hiring and preservation cost of quality employees and enable to establish the attrition position of 
www.rspsciencehub.com

employee by using the appropriate data extracting methods.

\section{Setiawan (2020)}

I Setiawan et.al done the research on "HR Analytics-Employee Attrition using Logistic Regression."

Researchers used Logistic Regression for analysing the employee attrition. For more understanding the authors apply $\mathrm{R}$ studio for data mixing, empirical data analysis, data planning, logistic regression, model assessment, and visualization.

Authors created 5 stages of their study - data gathering and business awareness, data premanaging, experimental data analysis, model selection and preparation, and analysis \& assessment of the model.

Employee attrition is studied with the help of variables as - number of companies operated, total work-experience, years with current supervisor, frequent professional travel, poor work environment satisfaction, department HR, marital status - separation, marital status - wedded, poor job satisfaction, early logout, and working overtime.

The Employee one of the main reasons, and The Company reason were also used. It is clear from the study, the retention rates, the company needs to develop the human resource department by assessing the working atmosphere, work or job satisfaction, amount of work of employee, and communication between manager, leader and subordinates.

\section{Christopher Boomhower (2018)}

Christopher Boomhower et.al researched and knowing us the "Employee Attrition-What makes an Employee Quit."

The study attempts to understand the underlying reasons for employee quit with special reference to civil workers. The authors examined publicly available data from office of Personnel Management, the Bureau of Labour Statistics, and IBM.
The researchers applied the principal Component Analysis methodology for exploring the reasons of attrition. The findings of the study, that pay scale is one of the major reasons for quitting the job.

The significant highlights of the study shows that, significant reduction in chances of an employee resigning as his or her service length increases, chances increase or decrease dependent on employee age, and chances of leaving are low if the employee is in the specification pay plan.

\section{Bradley E. Wright (2010)}

The research focus to evaluate the co-relation between job attraction-selection \& attrition with help of a model. The authors have considered the Public Sector for the study and studied public service motivation between private and public sector lawyers. The other important into understand the influence of PSM and how PSM influences employee sector choice.

The data gathered by the American Bar Association (ABA) to analyse the employment trends of lawyers. Authors designed survey and participants for more understanding and Panel Respondent Demographics by Sector of Employment for testing the job attraction selection - Attrition model.

Researchers concluded, relationship is not straightforward between PSM and sector employment. Even though the data used in this research do not rule out the opportunity that these answers are due to adaptation rather than magnetism-selection procedures, these results do not fully support either mechanism's opportunities that employee reward preferences will accord with the purpose each sector assists.

\section{Eric W. MacIntosh (2009)}

The objectives of the present research are to study the Impact the culture of the organization and job pleasure or fulfilment and intention to resign or quit in fitness industries. "The Cultural Index for Fitness Organizations (CIFO)" was established to evaluate views of organizational culture in the fitness industry. 
To understand the significance of fitness organizations, researchers conducted interviews with many fitness company managers, leaders, and experienced staff. The data was collected by administering the questionnaire with 7-point lid kart scale.

The Research included 11 dimensions namely: presence of organization, successful members, connections, reinforcement, innovation, marketing-sales, organizational reliability, health and ability, maintenance, work ethic, environment. Experimental factor analysis is employed which discovered eight components that characterize common dimensions of culture to this context: staff proficiency, ambience, connections among employees, validation, sales, service-gear, servicesystems, and organizational existence.

Organizational culture proportions, job pleasure and intention to quit developed by using path analysis.

The outcomes of the research showed $14.3 \%$ of the difference in job fulfilment and $50.3 \%$ of the difference with intention to quit the organization. It was clear that, multi-characteristic of organizational culture and its complication in the fitness industry.

\section{Romila Singh (2013)}

Authors have used Social Cognitive Career Theory Model to predict the turnover intentions among women employees.

SCCT model is used to predict the correlation among outcome opportunities, select career goals, effective actions, benefits, self-ability, as they might play the role for developing career. For further understanding, researchers used STEM theory and found the movement of women's representative in engineering and physical sciences. Also, they came to know about their intentional declination from post-degree and the occupation. (Ref: NSF, 2012; Preston, 2004; Society of Women Engineers, 2007).

Authors developed 6 Hypothesis and predicted that - Job mindsets, Relationship between social intellectual influences and job attitudes, Self- ability will facilitate the relationship between progressive opportunities at work and job position or job attitudes. Job Attitudes and Self-ability positively corelated with each other, Job attitudes should be related with Outcome opportunities or expectations. Outcome expectations will facilitate the correlation between growth opportunities at employment and job attitudes. Self-ability and outcome prospects will build the correlation between growth opportunities at workplace and job attitudes.

\section{Kashyap Bhuva (2018)}

Kashyap Bhuva et.al (2018) done the research on Machine Learning Techniques for Forecasting the Employee Attrition rate.

Researchers considered IT organization and collected the sample of the employee database of IBM USA.The current study is based on a workflow for the analytics project and considered segments - 1) Raw Dataset 2) Data Processing 3) Feature Selection \& Scaling 4) Modelling 5) Model Evaluation \& Tuning 6) Deployment \& Monitoring

Index-Terms-Ridge, Lasso, Logistic Failure, Judgmental structure, Random Forest, straight Discriminant Analysis, Support Vector System analytical techniques are the analytical tools used in the research.

Researchers predicted the attrition of IBM, USA data having 35 data mining techniques and machine learning algorithms by using different algorithms and combinations of several target attributes. The authors found, why intelligent and effective employee attrition prediction using data mining as Employee Attrition is one of the biggest Business Problem. Concluded the result of the prediction by data mining techniques with the similar dataset exposes that Linear Discriminant Analytical result beats than other one followed by Logistic Regression Model for a particular dataset if exactness is the metric favoured.

\section{Louis Lévy-Garboua (2008)}

The researchers done their research on Job Satisfaction and Resignations and used the wealth 
www.rspsciencehub.com

maximization theory of quitting behaviour on the German Socioeconomic Panel (1985-2003). Authors observed, job satisfaction is one of the most important reasons for employees who has exceptionally good experience and competency.

Researchers considered Voluntary Retirement, Satisfaction level from the job, Surprises for the employees, Wealth Maximization model for explaining the research. Authors explained the reasons for quitting, an employee's tendency to leave or resign from the organization must be comparable with the present values of future results and the outside opportunities compare to current job.

The results confirmed that through a simple subjective survey questionnaire about work structure, satisfaction level - Economists can draw or found plenty of hidden information or evidence easily. In-fact through this survey, a firm or industry can gain a prospect to improve them instead of using any traditional tools.

\section{Rupesh Khare (2019)}

Rupesh Khare et.al conducted their research on Employee Attrition Risk Assessment using Logistic Regression Analysis and they applied logistic regression technique and predicted employee attrition probability in an organization which is dependent on demographic data of divided employees.

Researchers executed the research based on real life project and collected demographic information from separated and existing employees. The questionnaire authors prepared, evaluate quitting probability with current staffs. The authors divided overall Attrition Risk into two part 1) Demographic Risk and 2) Behavioural Risk.

In the present study, based on demographic information, Logistic Regression mainly utilized to predict employee attrition risk. Along with this a proper retention plan has been mapped to focus on the risk categories developed.

\section{Resham Sundrani (2019)}

Volume 02 Issue 10S October 2020

The Author, Reshma Sundrani(2019), done her research on "Study on Employee Attrition \& Retention Exploring the Issues and Challenges". The research investigated \& considerable economical and intangible costs correlated with losing, expert and competent employees.

Researcher observed, the best practices of $\mathrm{HR}$ must be reflected in the organization and organizational policy which are related to employees. The HR strategy should grow from a transactional support role to partnering in the organizations business strategy.

\subsection{Employee retention strategies highlighted in the study are}

a) If organization acts as a source of pride and affiliation, $b$ ) When they respect their supervisors, c) If they are compensated and d) When they perceive their work as meaningful

The Researcher concludes that, HR policies, strategies and programs should go hand in hand along with the Vision and Mission of the business. Budget controlling measures that include cost cutting can only create a negative sentiment if employee satisfaction is overlooked.

\section{Objectives}

1. To understand the importance of HR Analytics in key performance areas.

2. To understand the role of HR Analytics in controlling attrition rate.

3. To know the various HR Analytical tools employed in organizations

\section{Input-Process and Output Framework of Attrition Reduction}

HR Plays a vital role in the organization by providing competent, skilled, experienced employees. An effective HR team of an organization always take care of their employees and build a healthy organizational environment, employees love to work for long term. Retention is most challenging job for an organization but if the employees get a healthy working environment with the proper compensation, appreciation and 
rewards they stay in the organization for long time. Now HR Analytics works on the pattern or trends of the organization and found the gaps where the organization needs the improvement(e.g. [12]) Like other challenges attrition one of the biggest challenges for any organization and HR analytical team can reduce the attrition by taking the proper steps from the beginning when the recruitment team hire employees for the organization.

In many cases we have seen after joining, employees didn't enjoy their job and within few months or years, they prepare their mindset for giving the resignation and here the team HR Analytics can make a change by using some input, process and output. Here we have created a model and selected specific objectives, input, process, and output. For reducing the attrition through HR Analytics, we considered the flow from beginning from the point recruitment starts.

\subsection{Recruitment:}

Human Resource planning most important task which enable the association to earn its strategic goals with knowledgeable, skilled, and experienced workforce. But for better fitment and employee retention, HR should explain the job description during their hiring process so that the candidates will have an idea, where they are going to join and what are the job responsibilities they need to take. Now for understanding the mind of the candidates HR needs to prepare proper recruitment framework so that they will be able to predict and able to read the candidate's mind as they want to work for long or short time.

\section{Process $\quad$ Outcome}

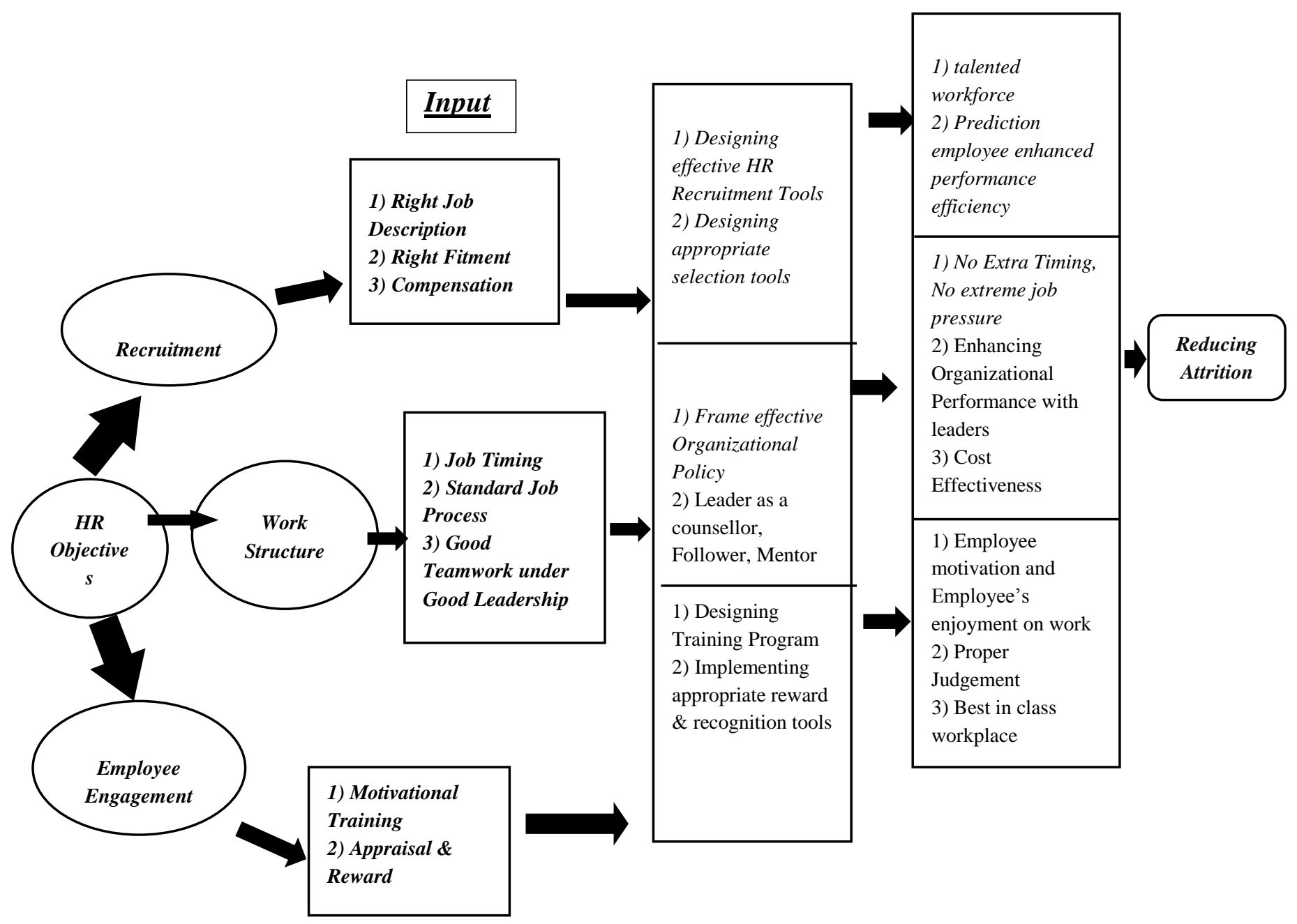

Model: Input-Process and Output Framework of Attrition Reduction 


\section{www.rspsciencehub.com}

Because Job satisfaction is very much important and that it only possible if the employees have clear idea about the job before they join in the organization. Along with the job satisfaction the HR recruiting team should be able to hire right fitment for a specific job. On the other hand, If the employees get the proper compensation, appreciation, and rewards, they will be happy to work in the same organization for long run. Hence, knowledge \& application of appropriate HR Analytical tools can provide useful information to the managers.

So, by proper recruitment process the best applicants would attest to be best strategy to the organization if the candidates joined and build long run relationship with the organization.

But if the organization looking for best outcome like Effective Recruitment \& Job Fitment, Proper Compensation for deserve Candidates, the HR Analytical Team need to Design effective HR Recruitment Tool, and appropriate selection tools. Because once HR practices enable to recruit competent employees and able to make them happy by providing a healthy working organization, the attrition rate will be reduced positively.[12-16]

4.2 Work Structure: Along with the effective recruitment process, Job Fitment and Compensation we have observed that proper working structure is also very much important for retaining the employees long run (Lévy-Garboua, Louis [9]) Our research says if anemployee overloaded with work and not getting any time for personal life \& family, after certain time will get frustrated and become demotivated. That is the very crucial moment for any employee for submitting their resignations. HR Analytical team has the abilities to work in those areas and able to predict the situation in an advance by framing proper organizational policies where no employee will be overloaded by their work and will be take extreme work pressure.

Manager's and Leader's behavior on employees also impact on the attrition rate. So as per the requirement organization needs to arrange some motivational, and counseling programs for the leaders. As an outcome the employees will not face to work extra time or no extreme job pressure.
Volume 02 Issue 10S October 2020

Through this organization will be able to enhance the performance of the employees as well.

\subsection{Employee Engagement: Lastly an} organization has responsibilities for giving a healthy and peaceful working environment to the employees so that the employees can work with full of happiness(ISetiawan[2]). More-over deserve employees should get proper appreciation, rewards, increment, bonus, incentives, and all. HR team need to investigate the matter so that organization able to do proper judgement. Considering the employee engagement, the HR analytical team can prepare effective framework for motivational training, rewards ceremony, effective tools for enhancing the knowledge of the employees.

With the application of proper HR Analytical tools, the attitude \& the employee behavior can be analyzed that intern can provide valuable insights to the HR Managers to take the right decisions.

\section{Implications for HR Analytics}

To participate in the dynamic world-wide environment, HR Analytics is the most essential technique of the industry. HR should show their interest to design (SDPs) analytical tools, frameworks to reduce the gaps at the micro level and enhance retention of the employees. Strategical movements and decisions play an important role to manage the changing aspects of the environment. Renovation, HR Analytical tools, frameworks, and methodology are managed and achieved the big players for improving overall improvement of the organization. Proper recruitment and assortment, right fitment is demanding the foremost attention to place proper talent with proper job satisfaction.

Now in the next step, most significant implication for the HR, design the effective framework for organizational policies for providing an effective and a suitable work structure to the employees. On the other hand, HR should design an effective counselling framework through which employees can discuss about their personal and professional stress, any discomforts, grievances etc. Healthy working environment always helps to enhancing 
the performance of the employees. Recognition and retention of employees are the need of the hour in the current competitive world. HR team of the large firm or industry can preserve their skilled, experienced, knowledge employees by maintaining of monitory and non-monitory elements of HR system. Here most important implications are modelling effective, motivational training and development program for employee engagement. Moreover, implementing proper rewards and recognition tools for proper judgement for competent, skilled, and talented employees is also equally essential. Considering the HR objectives, if HR takes the initiatives for implementing effective HR Analytical tools in the organizations that will positively help to reduce the attrition rate where others need to face difficult challenges as well.

\section{Conclusion}

HR Analytical tools can create \& exponential differentiation in areas as state as your statement

1. Revealed in employee turnover.

2. Time taking for hiring employee is for right fitment.

3. Investment is required for the employees for enhancing the productive speed

4. Need to find out the reasons of employees for leaving the organization within a year.

5. Employee Engagement program initiatives having an impact on employee performance and reducing attrition.

Several organizations recognized that extremely high cost is involved for replacing an employee and which almost over $200 \%$ of their annual remuneration, "AmericanProgress.org." described that the actual cost might even be higher due to training program / onboarding process(e.g. [15]). So, enhancing the retention and employee commitment are frequent to priorities for HR department. HR Analytics enable to help by improving the retention through a combine analysis that looks at data points. On the other hand, we have found that not only a single factor or objective is responsible to the attrition of any organization. There are several objectives which might be the reasons for the high attrition rate and HR Analytics can make different tools or frameworks for working on those objectives for reducing the attrition rate. In the current market scenario, we found that many big industries use specific HR Analytical tools for their organizations and by using those tools they improve the performance of the employees, finding out the gaps of the organization, reducing the attrition and improve overall organizational strategy.

\section{References}

[1]. Yadav, Sandeep, Aman Jain, and Deepti Singh. "Early Prediction of Employee Attrition using Data Mining Techniques." 2018 IEEE 8th International Advance Computing Conference (IACC). IEEE, 2018.

[2].Setiawan, I., et al. "HR analytics: Employee attrition analysis using logistic regression." IOP Conference Series: Materials Science and Engineering. Vol. 830. No. 3. IOP Publishing, 2020.

[3].Frye, Alex, et al. "Employee Attrition: What Makes an Employee Quit?" SMU Data Science Review 1.1 (2018): 9.

[4].Wright, Bradley E., and Robert K. Christensen. "Public service motivation: A test of the job attraction-selection-attrition model." International Public Management Journal 13.2 (2010): 155-176.

[5]. [MacIntosh, Eric W., and Alison Doherty. "The influence of organizational culture on job satisfaction and intention to leave." Sport Management Review 13.2 (2010): 106-117.

[6].Singh, Romila, et al. "Stemming the tide: Predicting women engineers' intentions to leave." Journal of Vocational Behaviour 83.3 (2013): 281-294.

[7]. Brown, Larry K., et al. "Predictors of retention among HIV/hemophilia health care professionals." General hospital psychiatry 24.1 (2002): 48-54.

[8]. Bhuva, Kashyap, and Kriti Srivastava. "Comparative Study of the Machine Learning Techniques for Predicting the Employee Attrition." IJRAR-International Journal of Research and Analytical Reviews (IJRAR) 5.3 (2018): 568-577.

[9].Lévy-Garboua, Louis, Claude Montmarquette, and Véronique Simonnet. 
"Job satisfaction and quits." Labour Economics 14.2 (2007): 251-268.

[10].Khare, Rupesh, et al. "Employee attrition risk assessment using logistic regression analysis." Ahmedabad: Indian Institute of Management. (2011).

[11].Norris, Pippa, ed. Passages to power: Legislative recruitment in advanced democracies. Cambridge University Press, 1997.

\section{Hyperlinks}

[12].https://www.valamis.com/hub/hranalytics\#: :text=HR\%20analytics $\% 20$ is $\%$ 20the $\% 20$ process, analytics $\% 2 C \% 20$ or $\% 20$ even $\% 20$ workforce $\% 20$ analytics.

[13].https://www.hrtechnologist.com/articles/h r-analytics/what-is-hr-analytics/

[14].https://www.questionpro.com/blog/hranalytics-and-trends/

[15].https://theeducationpaper.com/why-hranalytics-is-quintessential-fororganizations-now-dr-gireesh-y-msittumakuru/

[16].https://www.analyticsinhr.com/blog/hranalytics-tools/ 